\title{
Non-Invasive Glucose Monitoring: A Review of Challenges and Recent Advances
}

\author{
Tamar Lin*, Avner Gal, Yulia Mayzel, Keren Horman and Karnit Bahartan \\ Integrity Applications Ltd., Ashdod, Israel \\ Submission: July 17, 2017; Published: July 31, 2017 \\ *Corresponding author: Tamar Lin, Integrity Applications Ltd., 19 Ha’Yahalomim st. Ashdod, Israel, Tel: 9728675-7878; \\ Email: tamarl@integrity-app.com
}

\begin{abstract}
Glucose monitoring devices represent an exciting frontier in diabetes research. Great efforts have been dedicated to the development of non-invasive glucose monitoring devices, which may considerably improve the quality of life for people suffering from diabetes and facilitate their compliance for glucose monitoring. This manuscript reviews past, current and emerging non-invasive glucose monitoring techniques and devices and presents the major challenges they face. Poor glucose specificity and sensitivity, physiological time lag, calibration process and human factors perspective are discussed. Since incorporating user requirements into device development may potentially increase user acceptance and improve patient safety and device effectiveness, special attention is given to usability, user experience and applicability for home use, thus extending previous published reviews. The main conclusion is that a favorable non-invasive glucose monitoring device must overcome the combination of accuracy, usability and applicability challenges. Improving the assessment of device usability and acceptance may shed light on primary user concerns and may thus offer a meaningful step forward in the commercialization of non-invasive glucose monitoring devices.
\end{abstract}

Keywords: Glucose self-monitoring, Diabetes, Non-invasive glucose monitoring

Abbreviations: FDA: Food And Drug Administration, CEG: Clarke Error Grid, CE Communauté Européenne, Cgms Continuous Glucose Monitoring Systems, ISF Interstitial Fluid, Leds Light Emitting Diodes, MARD Mean Absolute Relative Difference, MU Main Unit, PEC Personal Ear Clip, RNIR Red/Near-Infrared, SNR Signal-To-Noise Ratio

\section{Introduction}

Glucose monitoring devices represent an exciting frontier in diabetes research, holding the potential to improve the lives of over 400 million people worldwide this number is expected to rise by approximately 55\% within the next 25 years [1]. During the past few decades there has been an increased need for an economic, compact, painless and convenient non-invasive device which can alleviate the pain associated with the frequent skin pricking and promote frequent glucose testing that helps in control of blood glucose levels [2]. Therefore, great efforts are made by researchers to develop such devices $[3,4]$. Ideally, these technologies should be painless, compact, lightweight, portable and easy to operate. At the same time they should provide reasonably accurate estimations of glucose concentration that are sufficient for critical behavioral, nutritional and drug dosing treatment decisions. Clearly, this is an extremely challenging mission.

State of the art developments in glucose monitoring include minimally invasive techniques using body fluids other than blood e.g., sweat, saliva, interstitial fluid ISF, compromising the skin barrier without puncturing blood vessels and non-invasive techniques, that mostly rely on optical methods [5]. Minimally invasive devices that monitor glucose continuously and automatically known as continuous glucose monitoring systems [CGMs] are already available in the market [6]. Non-invasive technologies are aimed to further advance this field by providing means to monitor glucose levels without pricking the skin barrier. These devices can either enable continuous readings similar to CGMs or intermittent readings where users actively perform measurements. During the past two decades great deal of efforts were dedicated to the development of non-invasive glucose monitoring devices. However, most of non-invasive technologies did not meet the required standard of accuracy and failed to run for a durable period of time [6].

This review presents the key challenges that the development of non-invasive devices is facing and discuss the methodologies addressing them. Several reviews have already dealt with this issue, however they lack information regarding, usability, user experience and applicability for home use. Integrating these 
factors in the device design has the potential to increase user acceptance and result in more successful products that improve patient safety and device effectiveness [7]. Therefore, a human factor perspective should be incorporated to the assessment of past, current and emerging non-invasive glucose monitoring devices.
In this review, any technique that does not involve drawing any human fluid sample was considered as a non-invasive glucose measurement approach. This paper complements conventional reviews which are based on scholarly published papers in journals. Special attention is given to products that have a Communauté Européenne CE Mark approval.

\section{Non-invasive technologies}

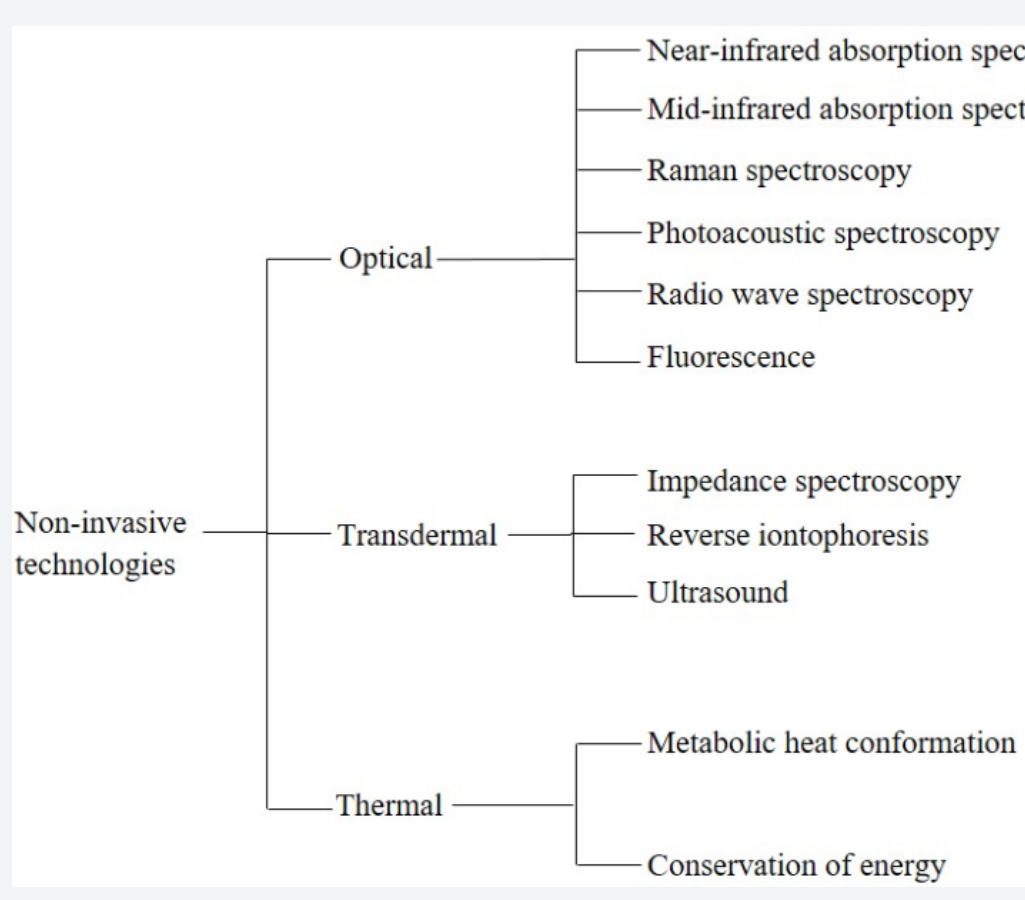

Figure 1: Non-invasive glucose measurement techniques.

The technologies used for non-invasive glucose monitoring include optical $[3,8]$, transdermal [4] and thermal techniques $[9,10]$ Figure 1. Optical techniques utilize the different properties of light to interact with glucose in a concentration-dependent manner. Transdermal techniques involve the measurement of glucose through the skin using either electricity or ultrasound. Finally, thermal techniques aim to measure glucose by detecting physiologic indices related to metabolic heatgeneration. Previous reviews provide detailed information on the advantages and limitations of each technique [3,4,11-13], thus in this review an emphasis is laid on the prospects and limitations of the devices derived from them. Briefly, transdermal techniques are easily affected by environmental variables e.g. temperature and sweat [13], while a major limitation of optical technologies is that they depend on the properties of the investigated tissue such as skin color tone [14].

\section{Non-invasive glucose monitoring devices}

Table 1 summarizes the information on past, current and emerging devices.

GlucoWatch ${ }^{\circledR}$ G2 Biographer: GlucoWatch G2 Biographer Cygnus Inc, USA was the first commercial device that was registered as a non-invasive glucose monitoring device and is the only product known as 'non-invasive' that was approved by the Food and Drug Administration FDA. However, the device was based on extraction of interstitial fluid through the skin, which is considered minimally invasive. Since it was registered as noninvasive the device was included in this review. In any case, GlucoWatch has an important role in the development of noninvasive technology, thus should not be disregarded.

GlucoWatch was a wristwatch-type device that provided real-time measurements of interstitial glucose concentrations at 10-minutes intervals. The device was intended for use by adults and children with type 1 or type 2 diabetes. The device utilized reverse iontophoresis to measures the amount of glucose molecules collected through a cathode disk with a sensor that contains the enzyme glucose oxidase [15]. The device had to be calibrated to account for skin permeability differences. However, this product was withdrawn from the market due to skin irritation and poor accuracy resulting from movement, exercising, sweating or rapid temperature changes.

Pendra ${ }^{\circledR}$ : Almost two decades ago Pendragon Medical Switzerland introduced Pendra, a continuous non-invasive device in the form of a wrist-watch that relied on impedance 
spectroscopy. The device required calibration and errors occurred due to sweating and motion. A post-marketing validation study revealed poor accuracy, with $4.3 \%$ readings out of 139 paired values in error zone $\mathrm{E}$ of the Clarke error grid CEG and a mean absolute relative difference of 52\% [16], resulting in the withdrawn of the device from the market. However, the intellectual property portfolio of this device is currently held by Biovotion AG, Zurich Switzerland, which is developing a multisensory concept for continuous non-invasive monitoring of health parameters.

OrSense NBM-200G: OrSense Ltd. Israel has developed the OrSense NBM-200G device for continuous glucose monitoring in people with type 1 or type 2 diabetes. The device uses a non-invasive optical measurement platform, combined with a finger attached ring-shaped sensor probe. The probe contains light sources and detectors operating in the red/near-infrared RNIR spectral region. It also contains pneumatic cuffs that produce oversystolic pressure to occlude blood flow, which generates a dynamic optical signal for glucose monitoring [17]. The device requires calibration prior to use. Clinical trials conducted on 23 subjects 1671 points demonstrated that $95.5 \%$ of the device readings were in the CEG A and B zones and that the mean absolute relative difference MARD was $17.2 \%$ [17]. Although this product received a CE-approval, it has never been commercialized.

C8 MediSensors: The C8 MediSensors Optical Glucose Monitoring System CA, USA relied on Raman spectroscopy: a beam of light is shone into the skin and the resulting vibrations of glucose molecules are measured to give a glucose reading. This device performed continuous glucose measurement every 5 minutes. One of its main advantages is was the lack of the need for calibration against blood glucose values. The percentage of points in the A zone and in the A+B CEG zones were $53 \%$ and $92 \%$, respectively and mean absolute differences was $38 \mathrm{mg} /$ $\mathrm{dL} \mathrm{N}=30$ [18]. This device received a CE-approval, but no longer exists.

GlucoTrack $®$ : GlucoTrack $\AA$ Integrity Applications Ltd., Israel determines glucose levels using three non-invasive techniques: ultrasonic, electromagnetic and thermal. The device consists of a main unit MU and a personal ear clip PEC, where the sensors are located. These sensors measure specific ultrasonic, electromagnetic and thermal parameters of the earlobe tissue, which occur due to glucose-related shifts in ion concentration, density, compressibility and hydration of both cellular and extracellular compartments of the tissue $[19,20]$. The device is intended for people with pre-diabetes and type 2 diabetes and requires an individual calibration, which establishes a baseline for physiological changes and reduces the impact of quasi-stable factors of the earlobe tissue. A clinical trial conducted on 17 subjects 4,418 points showed that $98.0 \%$ of its readings are in the clinically acceptable A and B zones of CEG with $52.4 \%$ in the A zone. The MARD and median absolute relative difference were
$22.8 \%$ and $19.0 \%$, respectively [21]. This device has received a $\mathrm{CE}$-approval and is commercially available in several countries.

Combo Glucometer ${ }^{T w}$ : Cnoga Medical Ltd. Israel developed the Combo Glucometer intended for adults any person above 18 years old with type 2 diabetes. The device is based on four light emitting diodes LEDs. Due to the absorption, when the light passes the fingertip, the spectrum that is detected also changes. The processor analyzes the signal and extracts the bio parameters from the signal. The device must be calibrated prior to use. Although the device appears on the company's site and has received a CE-approval, no information regarding its commercialization is available. There is lack of information on device performance as well.

Sugar BEAT ${ }^{\text {тM}}$ : SugarBEA Nemaura Medical Inc., United Kingdom offers a needle-free, wireless CGMs, suitable for people with type 1 , type 2 and pre-diabetes. The device measures the amount of glucose present at 5-minute intervals by drawing a small amount of glucose from the ISF into a patch placed on the skin through the passing of a mild, non-perceptible electric current across the skin. Clinical results indicated a MARD of $14.05 \%, 75 \%$ of the data in zone $\mathrm{A}$, and over $98 \%$ of the data in zones A and B of the CEG. However, these results were not peerreviewed. This device has recently received a CE-approval and is anticipated to be launched in 2017.

Gluco Wise $^{\text {TM: }}$ GlucoWise ${ }^{\mathrm{TM}}$ MediWise Ltd., United Kingdom extracts glucose levels by transmitting low-power radio waves through a section of the human body, such as the area between the thumb and forefinger or the earlobe. The device is still under development, expecting pre-orders in late 2018. However, at present little is known about the accuracy of this device and whether it offers an advantage over other devices.

\section{Key challenges of non-invasive glucose monitoring}

The major difficulties in the development of non-invasive glucose sensors are associated with the indirect nature of the measurement and the inevitable calibration process. This may respectively result in reduced accuracy, low usability and diminished applicability for home-use that require much effort to overcome. Effective solutions to these barriers will represent a significant advance in this field.

\section{Accuracy-related challenges}

Aiming to provide an accurate estimation of glucose levels, non-invasive devices face several accuracy-related key challenges that need to be overcome.

\section{Poor glucose specificity and sensitivity}

The indirect nature of non-invasive approaches subjects them to suffer from a relatively low signal-to-noise ratio SNR, since the measured parameters may be affected both from physiological factors other than glucose as well as from external elements. Several reviews of past and current non-invasive 
technologies indicate that non-invasive assays require additional increase of the SNR $[3,4,11,12]$. Most non-invasive devices rely on optical technologies (Table 1), which typically suffer from low sensitivity and specificity to glucose, due to the very low signal produced by glucose molecules [22]. Transdermal technologies may also be subjected to low SNR, as in the case of Pendra, which suffered from poor accuracy that was attributed to interference by other processes in the body [16]. GlucoTrack attempts to

Table 1: Information regarding non-invasive glucose monitoring devices $[6,12]$.

\begin{tabular}{|c|c|c|c|c|}
\hline Device & Technology & Target Area & Frequency & $\begin{array}{l}\text { Regulatory Approvals } \\
\text { and Commercialization }\end{array}$ \\
\hline $\begin{array}{l}\text { Animas Technologies } \\
\text { (Cygnus Inc.), } \\
\text { GlucoWatch } ® \text { G2 } \\
\text { Biographer (USA) }\end{array}$ & Reverse iontophoresis & Wrist Skin & Continuous & $\begin{array}{l}\text { CE-approved (1999) } \\
\text { and FDA-approved } \\
\text { (2001); was withdrawn } \\
\text { from the market due to } \\
\text { poor accuracy and skin } \\
\text { irritation }\end{array}$ \\
\hline $\begin{array}{c}\text { Pendragon Medical, } \\
\text { Pendra® (Switzerland) }\end{array}$ & Impedance Spectroscopy & Wrist Skin & Continuous & $\begin{array}{l}\text { CE-approved (2003); } \\
\text { was withdrawn from } \\
\text { the market following } \\
\text { poor accuracy in post- } \\
\text { marketing validation study }\end{array}$ \\
\hline $\begin{array}{l}\text { OrSense, NBM-200G } \\
\text { (Israel) }\end{array}$ & Occlusion Spectroscopy & Fingertip Skin & Intermittent & $\begin{array}{l}\text { CE-approved (2007); was } \\
\text { never commercialized. }\end{array}$ \\
\hline C8 MediSensors (CA, USA) & Raman Spectroscopy & Abdomen Skin & Continuous & $\begin{array}{l}\text { CE-approved (2011); the } \\
\text { company was closed due } \\
\text { to financial difficulties }\end{array}$ \\
\hline $\begin{array}{l}\text { Integrity Applications, } \\
\text { GlucoTrack® (Israel) }\end{array}$ & $\begin{array}{c}\text { Combination of } \\
\text { Thermal, ultrasonic and } \\
\text { electromagnetic }\end{array}$ & Earlobe tissue & Intermittent & $\begin{array}{l}\text { CE-approved (2013); } \\
\text { available in certain } \\
\text { countries }\end{array}$ \\
\hline $\begin{array}{l}\text { Cnoga Medical, Combo } \\
\text { Glucometer (Israel) }\end{array}$ & NIR Spectroscopy & Fingertip & Intermittent & $\begin{array}{l}\text { CE-approved (2014); } \\
\text { no information } \\
\text { available regarding } \\
\text { commercialization }\end{array}$ \\
\hline $\begin{array}{l}\text { Nemaura Medical } \\
\text { SugarBEAT }{ }^{\mathrm{T} м}(\text { United } \\
\text { Kingdom) }\end{array}$ & Reverse iontophoresis & Arm, leg or abdomen & Continuous & $\begin{array}{c}\text { CE-approved } \\
\text { (2016); market launch due } \\
2017\end{array}$ \\
\hline $\begin{array}{l}\text { MediWise GlucoWise }{ }^{\mathrm{TM}} \\
\text { (United Kingdom) }\end{array}$ & Radio wave spectroscopy & $\begin{array}{l}\text { skin between the thumb } \\
\text { and forefinger or the } \\
\text { earlobe }\end{array}$ & Continuous & $\begin{array}{c}\text { In development and will } \\
\text { be available once clinical } \\
\text { trials are completed. The } \\
\text { company expect to start } \\
\text { taking pre-orders in late } \\
2018 .\end{array}$ \\
\hline
\end{tabular}

The inability to convert the promising results in controlled settings to sustained accurate measurements at natural settings is another major concern. Previous technologies, such as impedance spectroscopy used in Pendra and reverse iontophoresis used in Glucowatch, were subjected to errors that occurred due to sweating and motion $[3,8]$. In contrast, devices that apply an intermittent measurement approach rather than a continuous one, such as GlucoTrack $\AA$ and Combo Glucometer, might reduce the effects of these interfering factors.

\section{Physiological time lag}

The physiological time lag between blood and tissue glucose decreases the accuracy of indirect glucose monitoring. This has been a major issue previously confronted by CGMs [23-25]. The increase glucose specificity by integrating three independent glucose-related technologies. This parallel monitoring of more than one parameter using multi-technology system is aimed to overcome the lack of specificity of each technology, since combining different factors provide a more comprehensive evaluation of glucose by covering various physical and chemical tissue parameters. 
layers. Thus, the accuracy may be affected by a general bloodtissue time lag that depends on a total lag between ISF and blood. Based on previous investigations of CGMs demonstrating that the physiological delay can be algorithmically corrected [27], it is suggested that non-invasive devices that estimate glucose from the skin should also utilize such an approach. For example, an advanced algorithm that corrects the physiological time lag by accounting for meal time and its glycemic load was employed in GlucoTrack.

\section{Usability and applicability challenges}

With a clear recognition of the critical role of usability and human interface in medical devices [28], another major aim is to make non-invasive glucose monitoring devices user-friendly, highly suitable for a wide population and applicable for home use. This is a challenging goal that requires several additional barriers to be addressed.

Device calibration: The indirect nature of non-invasive measurements requires calibration against concurrent blood glucose values, which provides an estimation of glucose concentration. The calibration process is conducted prior to using the device, in order to minimize the impact of individual quasi-stable factors, such as tissue thickness and structure. Typically, this process consists of several paired invasive-noninvasive measurements in a varying frequency, depending on the device and the technology employed. Since calibration is considered a reason for discomfort [29], utilizing a short and simple calibration procedure is expected to increase device usability and user satisfaction. Most of non-invasive devices imposed a long and complex calibration process. For example, OrSense NBM-200G required a daily 3 hour calibration period consisting of four measurements taken every hour [17]. Another example is Pendra, which required a patient-tailored calibration procedure lasting 2-3 days [16]. An even more extreme case is the Cnoga combo Glucometer that requires 1-2 weeks calibration process that includes at least 200 invasive readings http:// cnogacare.co/portfolio-item/combo-glucometer/. GlucoTrack utilizes a shorter and simpler calibration process that includes only 3 invasive measurements and takes less than 30 minutes. Similarly, in Sugar BEAT, each patch requires calibration by a single finger-stick reading, followed by a 30 minute warmup period. These patches, however, must be replaced every 24 hours http://www.sugarbeat.com

Another desired goal in non-invasive glucose sensing is reducing the calibration frequency and even negating it completely. Yet, most non-invasive devices require frequent calibration: the OrSense NBM-200G device requires a 3 hour daily calibration [3] and Pendra required a 1-h equilibrations and single-point blood glucose calibration each time it was worn $[30,31]$. More recent devices have successfully reduced the calibration frequency: GlucoTrack requires calibration every 6 months due to PEC replacement [21] and the Cnoga combo Glucometer does not require any sensor replacement or additional calibrations. The frequency of sensor replacement in these two devices is superior compared to CGMs in which the sensors can only be used for six to seven days [6]. In general, it seems that current developers of non-invasive devices are more aware that the calibration process its duration, complexity and validity period might have an effect on acceptance rates and devote much effort to making it more user friendly and applicable for home-use.

Suitability for various people: In order to reach high efficacy, non-invasive devices should be suitable for a variety of users. This is challenging since most of the technologies used to indirectly estimate glucose suffer from interfering human factors such as skin characteristics [12]. This is indeed a major limitation of optical techniques, since the transmission of light at each wavelength is a function of thickness, color and structure of the skin, bone, blood and other material through which the light passes [32]. This also the case with impedance spectroscopy, a major drawback of Pendra was that it was found to be unsuitable for a large group of patients due to strongly varying properties of the skin and underlying tissue [16]. Conversely, clinical trials demonstrate consistent performance of GlucoTrack among people with diverse profiles. Likewise, it has been proposed that GlucoWise provides consistent readings across all people independent of age or skin type and color due to the sensors that have integrated nano-composite films which temporarily make the skin transparent to the radio waves when a measurement is initiated http://www.gluco-wise.com

Applicability for home use: Another crucial issue in the development of non-invasive devices is their applicability for home/office use, manifested in simplicity and ultra-portability while adopting a human factor perspective. Unfortunately, most companies offer limited information regarding this issue. Glucowatch provides an example of low usability: the electrodes needed to be in place for at least 60 minutes, which exceeded the patience of many users [33]. Likewise, GlucoTrack also suffers from reduced usability, since the device requires to wait several minutes before performing a measurement after a shower, physical activity or entering indoor from outside. In contrast, an example for the adoption of a user-oriented approach is the implementation of a mobile app that communicates with the GlucoWise device via Bluetooth to display and store glucose readings. Although the importance of usability is now well acknowledged, there is limited information on device usability and user satisfaction for most non-invasive glucose monitoring devices. Specifically, usability information could not be found for any of the devices but a few published papers regarding user satisfaction were found. Satisfaction Peer-reviewed evidence was found for GlucoTrack, with clinical trials showing that homeuse of the device results in high levels of subject satisfaction and abilities to use and understand the device [21]. In contrast, Glucowatch ${ }^{\circledR}$ received unfavorable parental and youths' 
responses, indicating it required substantial improvement before it can achieve high acceptance and widespread utility [34].

\section{Discussion}

Non-invasive glucose monitoring holds great promise for improving diabetes management. Successful development of reliable non-invasive glucose monitoring devices that can operate over sustained periods of time has been an elusive goal in diabetes management. This review presents non-invasive technologies and the devices they have been utilized in and discusses key challenges in the development of such devices.

Previous reviews typically describe the main non-invasive glucose monitoring technologies and devices, focusing on the technical developmental stages. This papers extends these reviews by providing additional aspects related to user needs in term of device applicability and user acceptance. These are important and challenging issues that may determine whether a device will clinically and commercially fail or succeed [7]. Specifically, incorporation of usability aspects such as comfort, maintainability, portability and ease of both use and learning may affect patient safety and compliance with non-invasive selfglucose monitoring. Despite this, little research has been done to examine usability, user satisfaction and user acceptance of past, current and emerging non-invasive glucose monitoring devices. Acknowledging the importance of these factors, it should be noted that accuracy issues must first be addressed to produce reliable devices that meet clinically acceptable standards. Nonetheless, the development of commercially successful devices requires manufacturers to adopt usability and applicability perspectives in early stages of development.

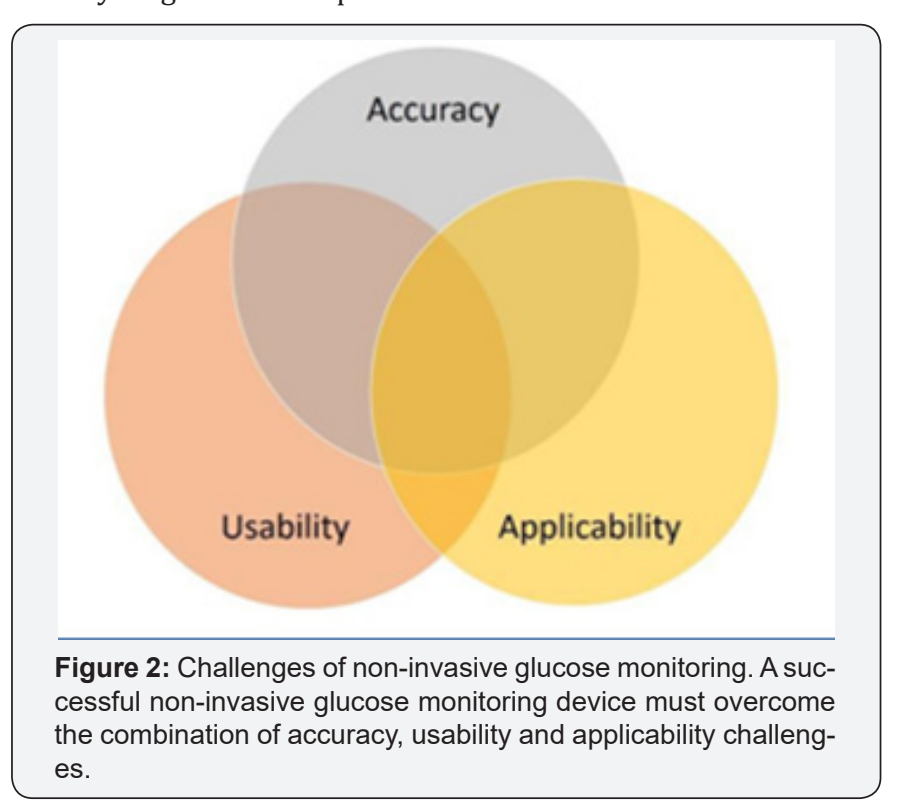

The main conclusion resulting from this review is that despite great efforts to make diabetes self-monitoring less invasive and cumbersome, many promising technologies that have been explored fail to overcome the tremendous challenges they face. The major challenges involved in the development of truly non-invasive glucose monitoring technologies are related to accuracy, usability and applicability for home use among a variety of people. Only a device that overcomes the combination of these challenges may provide a significant breakthrough in this field Figure 2. Such a device holds the potential to change the current gold standard use of invasive glucose biosensors and improve the quality of life of millions of people with diabetes worldwide. Currently, however, most devices still present a number of meaningful limitations, such as low sensitivity and specificity to glucose and the requirement of a complex and frequent calibration process. This indicates that the balance between generalizability and applicability is difficult to achieve. It is necessary to improve the assessment of device usability and acceptance, which may shed light on primary user concerns and may thus offer a meaningful step forward in the commercialization of these devices.

A major limitation of current non-invasive devices is that they cannot completely substitute a traditional glucose meter. Therefore, these devices must be under constant improvements, aiming to further advance performance by significantly improving the algorithms, software and device features. Additionally, further clinical studies are needed to determine whether utilization of non-invasive devices will indeed lead to enhanced glycemic control in patients using this technology. It should also be noted that the current review did not address the challenge of cost-effectiveness.Non-invasive glucose monitoring devices are also economically appealing for consumers as they do not require lancets or strips, however some of the technologies discussed require high cost [12].

\section{Conclusion}

Although there has been much research dedicated to develop a non-invasive glucose monitoring device, the complexity and indirect nature of the measurement process remain barriers to the successful development of truly non-invasive technologies implemented in user-friendly devices. Nonetheless, some of the devices discussed here have made considerable progress in recent years, introducing an exciting opportunity for self-glucose monitoring that offers an adequately accurate and simple way for painless and convenient self-monitoring of glucose levels. Yet, continuous efforts must constantly be made to further evaluate and improve performance, usability and user acceptance, and to clearly illustrate the benefits of using such a device.

\section{Conflict of Interest}

T.L, A.G, Y.M, K.H and K.B are/were employees of Integrity Applications, and may potentially own stocks or hold stock options in the company.

\section{References}

1. International Diabetes Federation (IDF) diabetes atlas (2015) Brussels, Belgium: International Diabetes Federation. 
2. Ong WM, SS Chua, CJ Ng (2014) Barriers and facilitators to selfmonitoring of blood glucose in people with type 2 diabetes using insulin: a qualitative study. Patient Prefer Adherence 8: 237-246.

3. Vashist SK (2012) Non-invasive glucose monitoring technology in diabetes management: a review. Anal Chim Acta 750: 16-27.

4. do Amaral CEF, B Wolf (2008) Current development in non-invasive glucose monitoring. Med Eng Phys 30(5): 541-549.

5. Govada A, C Renumadhavi and KB Ramesh (2014) Non-invasive blood glucose measurement. Int J Adv Res Comput Commun Eng 3(1): 5122 5125 .

6. Vashist SK (2013) Continuous Glucose Monitoring Systems: A Review. Diagnostics (Basel) 3(4): 385-412.

7. Martin JL, E Murphy, JA Crowe and BJ Norris (2006) Capturing user requirements in medical device development: the role of ergonomics. Physiol Meas 27(8): R49-62.

8. Oliver NS, C Toumazou, AEG Cass and DG Johnston (2009) Glucose sensors: a review of current and emerging technology. Diabet Med 26(3): 197-210.

9. Cho OK, YO Kim, H Mitsumaki, K Kuwa (2004) Noninvasive Measurement of Glucose by Metabolic Heat Conformation Method. Clin Chem 50(10): 1894-1898.

10. Zhang Y, J Zhu, Y Liang, H Chen, S Yin, Z Chen (2017) Non-invasive blood glucose detection system based on conservation of energy method. Physiol Meas 38(2): 325-342.

11. Aggidis AGA, JD Newman, GA Aggidis (2015) Investigating pipeline and state of the art blood glucose biosensors to formulate next steps. Biosens Bioelectron 74: 243-262.

12. Salam NABA, WH bin M Saad, ZB Manap, F Salehuddin (2016) The Evolution of Non-invasive Blood Glucose Monitoring System for Personal Application. J Telecommun Electron Comput Eng JTEC 8(1): 59-65.

13. Hatice VD, Y Tulay (2017) Noninvasive Glucose Measurement for Diabetes Mellitus Patients. Curr Trends Biomed Eng Biosci 2

14. Anderson RR, JA Parrish (1982) Optical Properties of Human Skin. In The Science of Photomedicine, Regan, JD and JA Parris (Eds.), Springer, pp. 147-194.

15. Tierney MJ, JA Tamada, RO Potts, RC Eastman, K Pitzer, et al. (2000) The GlucoWatch $\AA$ biographer: a frequent, automatic and noninvasive glucose monitor. Ann Med 32(9): 632-641.

16. Wentholt IME, JBL Hoekstra, A Zwart and JH DeVries (2005) Pendra goes Dutch: lessons for the CE mark in Europe. Diabetologia 48(6): 1055-1058.

17. Amir O, D Weinstein, S Zilberman, M Less, D Perl-Treves, H Primack, et al. (2007) Continuous noninvasive glucose monitoring technology based on"occlusion spectroscopy." J Diabetes Sci Technol 1(4): 463469.

18. Lipson J, J Bernhardt, U Block, WR Freeman, R Hofmeister, et al. (2009) Requirements for calibration in noninvasive glucose monitoring by Raman spectroscopy. J Diabetes Sci Technol 3(2): 233-241.

19. Harman-Boehm I, A Gal, AM Raykhman, JD Zahn, E Naidis, et al. (2009) Noninvasive Glucose Monitoring: A Novel Approach. J Diabetes Sci Technol 3(2): 253-260.
20. Harman-Boehm I, A Gal, AM Raykhman, E Naidis and Y Mayzel (2010) Noninvasive glucose monitoring: increasing accuracy by combination of multi-technology and multi-sensors. J Diabetes Sci Technol 4(3): 583.

21. Horman K, Y Mayzel, A Gal, K Bahartan, A Drexler and T Lin (2016) Performance and User Experience Evaluation of a Non-Invasive Glucose Monitoring Device. Int J Diabetes Metab Disord 1(2): 1-7.

22. Srivastava A, MK Chowdhury, S Sharma and N Sharma (2013) Blood glucose monitoring using non invasive optical method: Design limitations and challenges. Int J Adv Res Electr Electron Instrum Eng 2: $615-20$

23. Cengiz E and WV Tamborlane (2009) A tale of two compartments: interstitial versus blood glucose monitoring. Diabetes Technol Ther 11(1): S11-S16.

24. Stout PJ, N Peled, BJ Erickson, ME Hilgers, JR Racchini, et al. (2001) Comparison of Glucose Levels in Dermal Interstitial Fluid and Finger Capillary Blood. Diabetes Technol Ther 3(1): 81-90.

25. Groenendaal W, G von Basum, KA Schmidt, PA Hilbers, NA van Riel (2010) Quantifying the composition of human skin for glucose sensor development. J Diabetes Sci Technol 4(5): 1032-1040.

26. Keenan DB, JJ Mastrototaro, G Voskanyan, GM Steil (2009) Delays in Minimally Invasive Continuous Glucose Monitoring Devices: A Review of Current Technology. J Diabetes Sci Technol 3(5): 1207-1214.

27. Rebrin K, GM Steil, WP Van Antwerp, JJ Mastrototaro (1999) Subcutaneous glucose predicts plasma glucose independent of insulin: implications for continuous monitoring. Am J Physiol-Endocrinol Metab 277(3 Pt 1): E561-E571.

28. International Electrotechnical Commission (2007) Medical Devices: Application of Usability Engineering to Medical Devices (International Electrotechnical Commission).

29. Acciaroli G, M Vettoretti, A Facchinetti, G Sparacino, C Cobelli (2016) From Two to One Per Day Calibration of Dexcom G4 Platinum by a Time-Varying Day-Specific Bayesian Prior. Diabetes Technol Ther 18(5): 472-479.

30. Weinzimer SA (2004) Analysis: PENDRA: The Once and Future Noninvasive Continuous Glucose Monitoring Device? Diabetes Technol Ther 6(4): 442-444.

31. Caduff A, E Hirt, Y Feldman, Z Ali, L Heinemann (2003) First human experiments with a novel non-invasive, non-optical continuous glucose monitoring system. Biosens Bioelectron 19(3): 209-217.

32. Jagemann K-U, C Fischbacher, K Danzer, UA Mueller and B Mertes (1995) Application of near-infrared spectroscopy for non-invasive determination of blood/tissue glucose using neural networks. Z Für Phys Chem 191: 179-190.

33. So CF, KS Choi, TKS Wong, J Chung (2012) Recent advances in noninvasive glucose monitoring. Med Devices (Auckl) 5:45-52

34. Diabetes Research in Children Network (2005) Youth and Parent Satisfaction With Clinical Use of the GlucoWatch G2 Biographer in the Management of Pediatric Type 1 Diabetes. Diabetes Care 28: 19291935. 
Your next submission with Juniper Publishers will reach you the below assets

- Quality Editorial service

- Swift Peer Review

- Reprints availability

- E-prints Service

- Manuscript Podcast for convenient understanding

- Global attainment for your research

- Manuscript accessibility in different formats ( Pdf, E-pub, Full Text, Audio)

- Unceasing customer service

Track the below URL for one-step submission https://juniperpublishers.com/online-submission.php 